\title{
Revisión de técnicas microscópicas para modelos digitales de roca
}

\author{
Andrei Felipe Lozano ${ }^{*}$ (D); Tatiana Juliao-Lemus ${ }^{2}$; Edgar Ricardo Pérez $^{2}$; \\ Ayerim Antonieta Obando-Yaguas ${ }^{2}$
}

Forma de citar: Lozano, A.F; Juliao-Lemus, T.; Pérez, E.R.; Obando-Yaguas, A.A. (2022). Revisión de técnicas microscópicas para modelos digitales de roca. Boletín de Geología, 44(1), 161-171. https://doi.org/10.18273/ revbol.v44n1-2022008

\begin{abstract}
Resumen
Los yacimientos no convencionales (roca generadora) representan una nueva etapa en la exploración y explotación de petróleo y gas a nivel mundial, y su caracterización petrofísica sigue siendo un desafío, debido a las bajas permeabilidades, los altos niveles de heterogeneidad y la dificultad de adaptación de las técnicas convencionales. La petrofísica digital surge como una alternativa que aprovecha los últimos avances en la microscopía electrónica, la tomografía computarizada y el procesamiento computacional para, a través de métodos numéricos y algoritmos de conteo de vóxel, estimar las propiedades petrofísicas en lo que se denomina un modelo de roca digital. En este trabajo se realiza una revisión de las técnicas de caracterización digital y su aplicación en muestras de yacimientos no convencionales pertenecientes a la Formación Vaca Muerta (Argentina) y Formación La Luna (Colombia). Con esta tecnología es posible visualizar el espacio poroso a escala micro- y nanométrica, con el fin de obtener información cualitativa (tipos de poro y microfracturas) y cuantitativa (porosidad, permeabilidad absoluta, distribución de tamaño de poro, cantidad de materia orgánica y propiedades petrofísicas avanzadas). Los resultados obtenidos indican que las muestras FIB-SEM se encuentran por debajo del volumen elemental representativo y que las muestras digitales con mayores dimensiones, aunque más representativas, requieren de una mayor capacidad computacional. El escalamiento de las propiedades petrofísicas, la falta de conectividad del medio poroso y la baja representatividad son las principales limitantes presentes en la tecnología. Sin embargo, su potencial aumenta conforme la inteligencia artificial, la simulación y el machine learning toman fuerza en la industria del petróleo.
\end{abstract}

Palabras clave: Yacimientos de roca generadora; Shale; Petrofísica digital; Física digital de rocas.

\section{Review of Microscopic Techniques for Digital Rock Models}

\begin{abstract}
Unconventional deposits (source rock reservoirs) represent a new stage in the exploration and exploitation of oil and gas worldwide, and their petrophysical characterization continues to be a challenge due to low permeabilities, high levels of heterogeneity, and the difficulty of adapting conventional techniques. Digital petrophysics emerges as an alternative that takes advantage of the latest advances in electron microscopy, computed tomography, and computational processing to estimate petrophysical properties using numerical methods and voxel counting algorithms in what is called a digital rock model. This work carries out a review of digital characterization techniques and their application to unconventional reservoir samples belonging to the Vaca Muerta Formation (Argentina) and La Luna Formation (Colombia). With this technology, it is possible to visualize the porous space on a micro and nanometric scale to obtain qualitative information (types of pores and microfractures) and quantitative information (porosity, absolute permeability, pore size distribution, organic matter content, and advanced petrophysical properties). The results obtained indicate that the FIB-SEM samples are below the representative elementary volume and that digital samples with larger dimensions, although more representative, require greater computational capacity. The upscaling of petrophysical properties, the lack of connectivity of the porous medium, and the poor representativeness are the most important limitations of using this technology. However, its potential increases as artificial intelligence, simulation, and machine learning techniques gain strength in the oil and gas industry.
\end{abstract}

Keywords: Source rock reservoirs; Shale; Digital petrophysics; Digital rock physics.

${ }^{1}$ Escuela de Geología, Universidad Industrial de Santander, Bucaramanga, Colombia. $\left(^{*}\right)$ andrei.lozano@hotmail.es ${ }^{2}$ Centro de Innovación y Tecnología - ICP, Ecopetrol, Piedecuesta, Colombia. tatiana.juliao@ecopetrol.com.co, ricardo.perez@ecopetrol.com.co, ayerim.obando@ecopetrol.com.co 


\section{Introducción}

Las rocas presentes en los yacimientos no convencionales se caracterizan por su alta complejidad, baja porosidad $(1-15 \%)$ y permeabilidad $(<1 \mathrm{mD})$ y por la magnitud micro- y nanométrica (tamaño de grano $<6$ micras) de sus estructuras (Zoback y Kohli, 2019). Lo anterior dificulta la caracterización de la roca a nivel petrográfico y petrofísico, puesto que muchos de los instrumentos tradicionales fueron diseñados para trabajar con muestras de mejor calidad. La petrofísica digital surge como una alternativa que permite extraer información a partir de la adquisición de imágenes digitales de la roca en cuestión. Históricamente, las dos limitantes principales que impedían el desarrollo de esta tecnología eran la capacidad de producir imágenes en alta resolución y la habilidad de ejecutar operaciones de cómputo a una alta velocidad (Dvorkin et al., 2009). Actualmente, existen instrumentos que escanean digitalmente en alta resolución y clusters computacionales que permiten ejecutar procesos de simulación en tiempos económicamente viables. En consecuencia, desde el año 2007, proliferan compañías y universidades que ofrecen soluciones digitales al servicio de la industria petrolera.

En este trabajo, se analiza una muestra (prisma rectangular digital) de FIB-SEM de las formaciones Vaca Muerta (muestra \#1) y La Luna (muestra \#2), las cuales corresponden a una roca generadora tipo lutita. La muestra \#1 se obtuvo del portal digital de rocas de la Universidad de Texas (TACC, por sus siglas en inglés) y no se dispone de una descripción mineralógica; se compone de 600 imágenes de FIBSEM a una resolución de 2,5 × 2,5 × 2,5 nm por píxel (Andrew, 2019). La muestra \#2 fue proporcionada por Ecopetrol, pertenece al miembro Galembo de la Formación La Luna y se caracteriza por la presencia de un alto contenido de cuarzo (>70\%), un contenido de carbonatos de $14 \%$ y un contenido de arcillas de $9 \%$, donde la illita es la arcilla predominante; se compone de 264 imágenes de FIB-SEM a una resolución de $10 \times 10 \times 10 \mathrm{~nm}$ por píxel. Con el fin de comparar resultados de porosidad y materia orgánica obtenidos digitalmente con resultados de métodos convencionales de laboratorio, se utilizaron 13 imágenes BIB-SEM en diversas muestras de la Formación La Luna. Se analizan las limitantes encontradas y las consideraciones para tener en cuenta al momento de aplicar esta tecnología, con el fin de extraer información petrofísica a partir de imágenes digitales.

\section{Marco teórico}

Las técnicas de imagen de mayor potencial en la generación de modelos $3 \mathrm{D}$ de roca digital son la tomografía computarizada o macro-CT, la microtomografía o micro-CT y la microscopía electrónica de barrido SEM. Por cuestiones de resolución, las dos primeras técnicas (macro- y microCT) se aplican con mayor frecuencia en el análisis de rocas convencionales; para el análisis de roca generadora, con el fin de visualizar el medio poroso, se utiliza la microscopía de electrones y/o iones (FIBSEM, BIB-SEM y HIM) en lugar de la tomografía de rayos $\mathrm{X}$, puesto que las técnicas tipo SEM proveen imágenes con una resolución nanométrica. Aunque ya existen nanotomógrafos en el mercado, que alcanzan una resolución nanoescala (e.g. 30-40 nm por píxel), estos no permiten visualizar el medio poroso de la roca generadora en su totalidad, $\mathrm{y}$, por lo tanto, se utilizan principalmente en el monitoreo de la evolución de la materia orgánica en condiciones variables de temperatura (Lin et al., 2010; Saif et al., 2017), estudio de laminaciones y caracterización de fracturas naturales.

La microscopía tipo BIB-SEM (broad ion beam) permite realizar un desbastado inicial utilizando iones de argón para obtener superficies lo suficientemente lisas como para poder diferenciar adecuadamente las diferentes fases (minerales, porosidad y materia orgánica) presentes en la muestra por analizar (Klaver et al., 2012). Estas superficies son más representativas (tipo mosaicos) en el análisis de yacimientos en roca generadora, pero solo proveen una cuantificación bidimensional. Aunque no es completamente necesario aplicar esta técnica en la petrofísica digital de roca generadora, su implementación facilita la selección de zonas de interés en alta resolución y proporciona información adicional que puede ser utilizada durante la aplicación de metodologías de escalamiento de los resultados obtenidos.

El FIB-SEM (focused ion beam) utiliza iones de galio para generar una erosión de superficie (Figura 1) y la posterior composición de imágenes en un apilamiento volumétrico digital (Giannuzzi, 2004), el cual es utilizado para extraer, a través de un proceso de segmentación/ binarización, el medio poroso de la roca generadora en cuestión. Es una de las técnicas de imagen más aplicadas en el estudio de rocas no convencionales tipo lutita, debido a su aporte en la obtención de mineralogía con la posibilidad de generar mapas mineralógicos en tiempo real, con la implementación de múltiples detectores 
EDS (energy-dispersive X-ray spectroscopy) (Li et al., 2021), la diferenciación adecuada de fases minerales de imágenes obtenidas con BSE (backsattered electrons) y la caracterización cualitativa de imágenes SE (secondary electrons) y cuantitativa (aplicación de algoritmos de conteo de vóxel) de la porosidad de la roca.

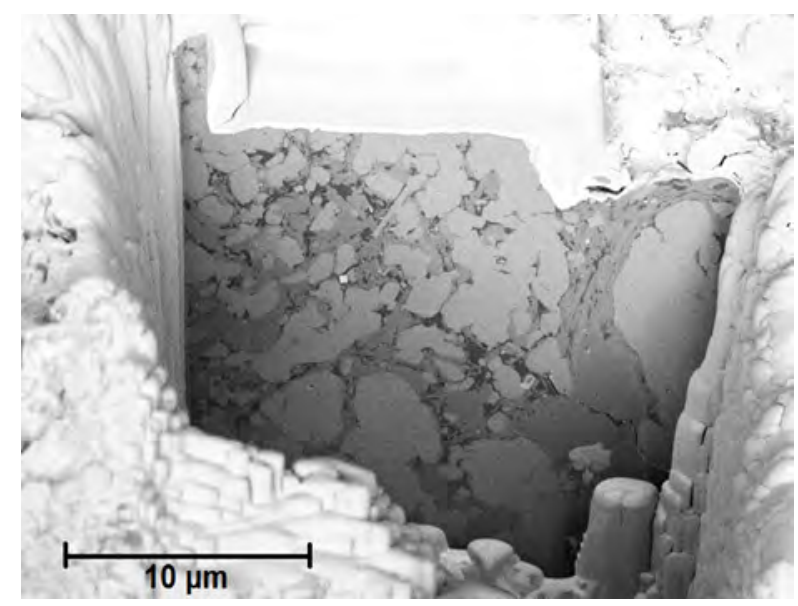

Figura 1. Proceso de adquisición de volumen FIB-SEM de alta resolución (10 nm/píxel) en muestra de la Formación La Luna.

Además, los últimos avances en petrofísica digital permiten la generación de modelos PNM (pore network modelling) y la segmentación de fase porosa y microporosa para alimentar modelos de propiedades petrofísicas especiales como la estimación de la permeabilidad relativa (Cantisano et al., 2013), la presión capilar por inyección de mercurio, y las propiedades eléctricas (factor de formación) y térmicas (conductividad termal) (Nozad et al., 1985), entre otras.

\section{Resultados}

\section{Caso de estudio: muestra \#1 Formación Vaca Muerta}

A partir del análisis de las imágenes FIB-SEM de la muestra \#1 Formación Vaca Muerta, se identificaron tres fases minerales y una fase de materia orgánica (bitumen/ kerógeno) (Figura 2B). La porosidad presente en la muestra se encuentra asociada con la materia orgánica, como se observa en la Figura 2A. La porosidad 3D obtenida del volumen digital es de 3,92\% (Figura 3A). Sin embargo, al calcular la porosidad 2D sobre cada imagen que compone el volumen digital, se observa que la porosidad relacionada con la materia orgánica varía entre 2,8-6\%, como se observa en la Figura 3B.
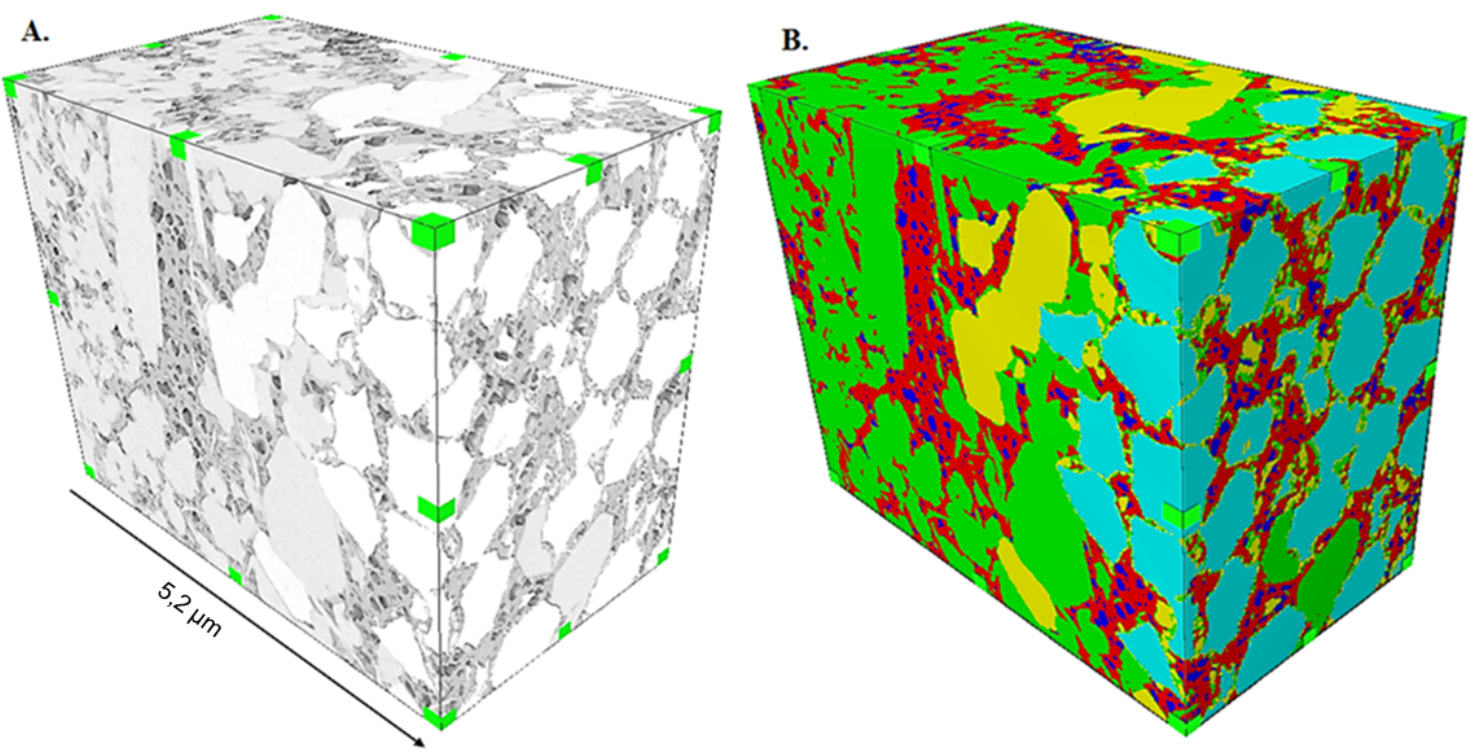

\begin{tabular}{|c|c|c|c|c|}
\cline { 2 - 5 } \multicolumn{1}{c|}{ C. } & Frac ción volumétrica & Vóxeles contados & Vóxeles totales & Fase segmentada \\
\hline 1 & 0,039 & 87.568 .066 & 2.232 .310 .080 & Porosidad 3D \\
\hline 2 & 0,291 & 506.199 .459 & 2.232 .310 .080 & Materia Orgánica \\
\hline 3 & 0,419 & 936.315 .071 & 2.232 .310 .080 & Mineral 1 Oscuro \\
\hline 4 & 0,094 & 210.489 .221 & 2.232 .310 .080 & Mineral 2 Claro \\
\hline 5 & 0,156 & 348.079 .427 & 2.232 .310 .080 & Mineral 3 Brillante \\
\hline
\end{tabular}

Figura 2. A. Aplicación del filtro non-local-means (FIB-SEM) y B. Segmentación de las fases minerales y porosidad presentes en la muestra \#1 Formación Vaca Muerta C. En la tabla se observa la fracción volumétrica de cada una de las fases segmentadas. 
A.

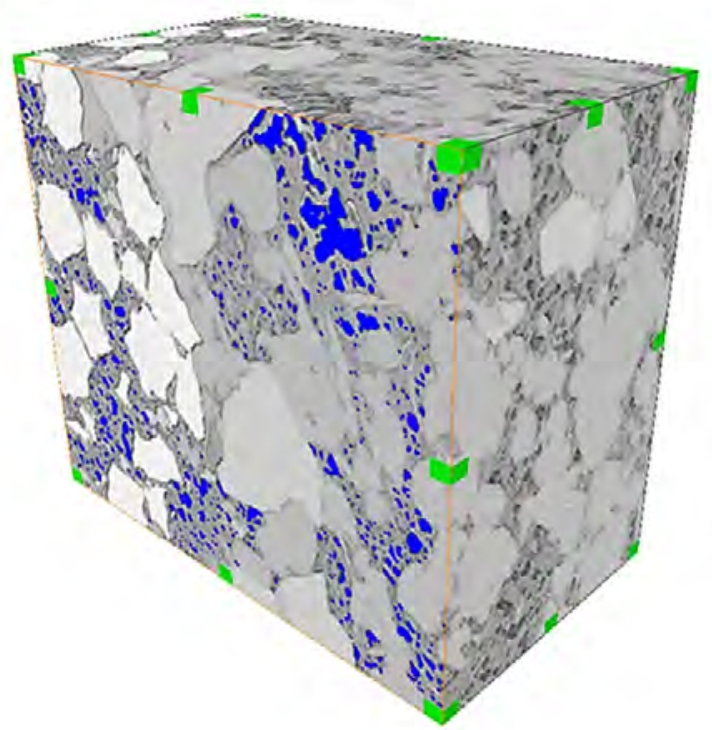

B.

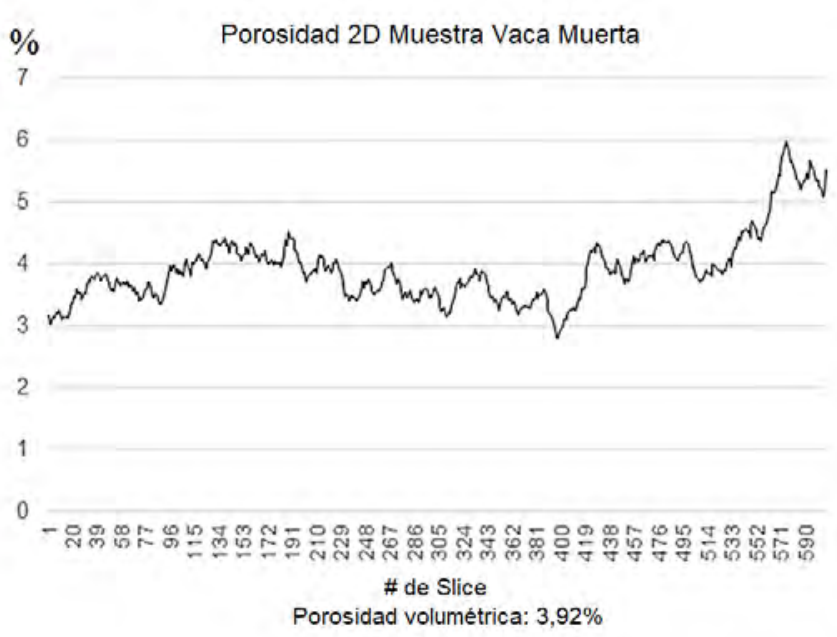

Figura 3. A. Estimación de la porosidad 3D en muestra \#1 Formación Vaca Muerta y B. Variación de la porosidad 2D a lo largo de cada imagen serial.

Con el fin de caracterizar el contenido de materia orgánica presente en la muestra digital, se utilizó el método de segmentación threshold (Al-Amri et al., 2010), seguido del método watershed (Levner y Zhang, 2007); posteriormente, se calculó un radio de transformación aparente (RTA) de 12\%, como se observa en la Figura 4, el cual es el porcentaje de materia orgánica que puede convertirse en hidrocarburos libres y porosidad (McLean et al., 2017).

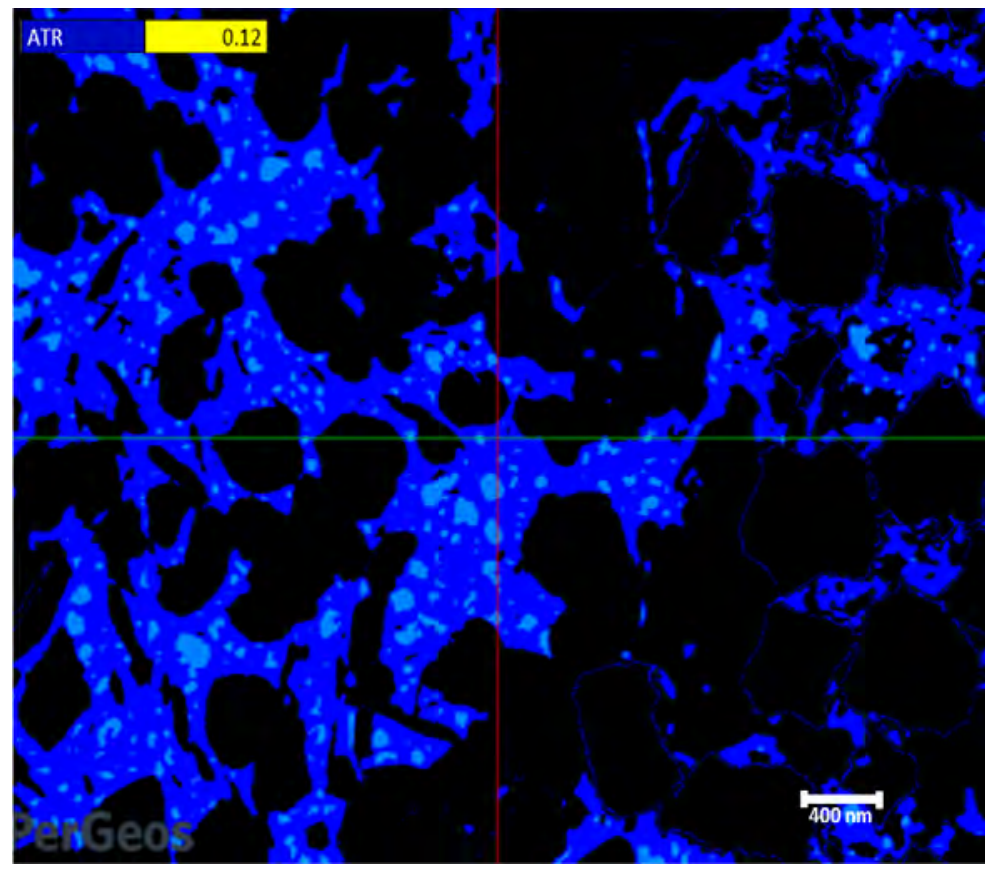

Figura 4. Cuantificación de contenido de materia orgánica y estimación de la tasa de transformación aparente en muestra \#1 Formación Vaca Muerta. En color azul claro se visualiza la porosidad relacionada con la materia orgánica, en color azul oscuro se visualiza la materia orgánica (kerógeno/bitumen) y en color negro se tienen las fases minerales que componen la muestra. 
La segmentación del espacio poroso relacionado con la materia orgánica permite realizar la medición de cada uno de los poros identificados en la imagen, con el fin de obtener la distribución de tamaño de poro presente en la muestra. Como se observa en la Figura 5, la muestra presenta una distribución unimodal, donde se obtiene una moda de $2 \mathrm{~nm}$.

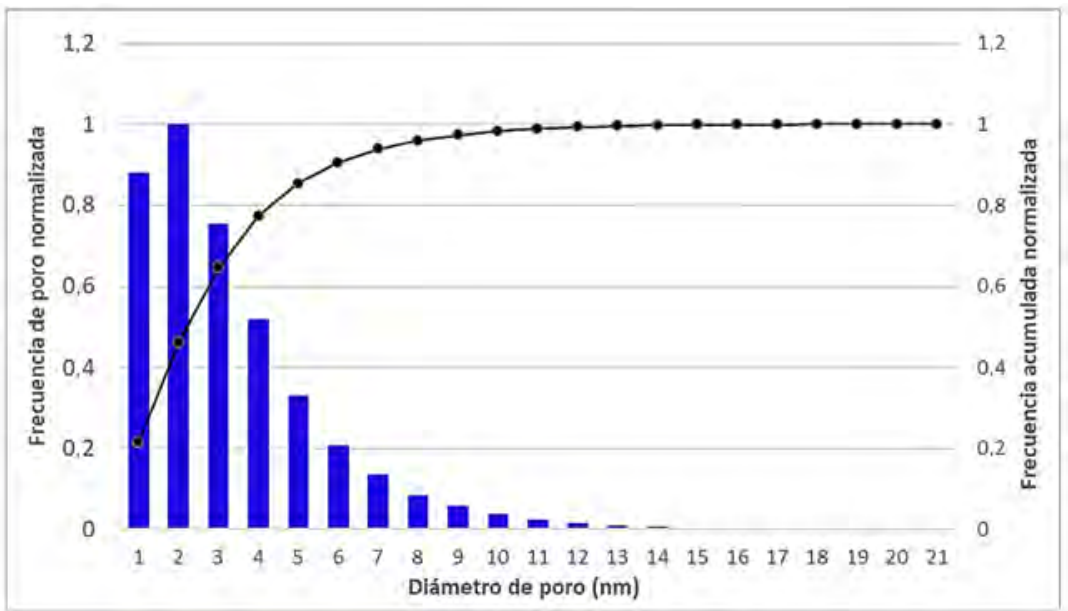

Figura 5. Estimación de la distribución de tamaño de poro en muestra \#1 Formación Vaca Muerta a partir de imágenes FIB-SEM.

Posteriormente, se identificó la porosidad conectada a lo largo del eje Y (Figura 6A), sobre la cual se aplicaron algoritmos de simulación de tipo volúmenes finitos (Chorin, 1967) que permiten estimar la permeabilidad absoluta (Figura 6B) de la muestra a partir de la solución de la ecuación de Stokes (Zhang et al., 2019):

$$
\begin{gathered}
\vec{\nabla} \cdot \vec{V}=0 \\
\mu \nabla^{2} \vec{V}-\vec{\nabla} P=\overrightarrow{0}
\end{gathered}
$$

Donde $\vec{V}$ es la velocidad del fluido, $\vec{\nabla}$ es el operador gradiente, $\mu$ es la viscosidad del fluido, $\nabla^{2}$ es el operador laplaciano y $P$ es la presión del fluido.

Durante la ejecución de la simulación, se utilizó una presión de entrada de $130.000 \mathrm{~Pa}$, una presión de salida de $100.000 \mathrm{~Pa}$ y una viscosidad del fluido de 0,001 Pa.s. Se obtuvo una permeabilidad absoluta de $828 \mathrm{nD}$ (Tabla 1).
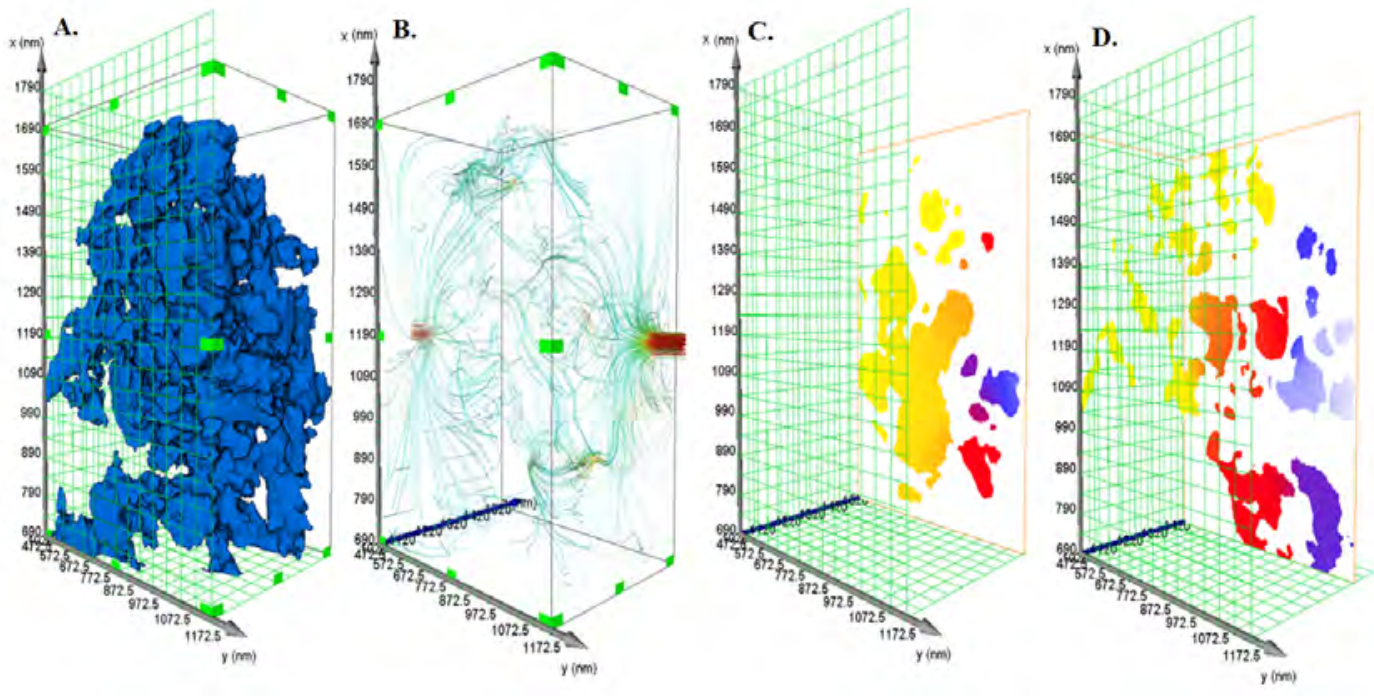

Figura 6. Muestra digital \#1 de la Formación Vaca Muerta. A. Segmentación de la porosidad conectada en dirección Y. B. Líneas iluminadas representando el campo de velocidad en el cálculo de permeabilidad absoluta. C. Visualización del campo de concentración en experimento de simulación de difusión molecular en dirección Y. D. Visualización del campo potencial en el experimento de simulación de flujo eléctrico en dirección Y. 
La porosidad conectada permitió aplicar la simulación de difusión molecular (Figura 6C), la cual se basa en la solución de la ley de Fick (van Milligen et al., 2005):

$$
\vec{J}=-D \cdot \vec{\nabla} c
$$

En donde $\vec{J}$ es el flujo difusivo $\left(\mathrm{mol} \cdot \mathrm{m}^{-2} \cdot \mathrm{s}^{-1}\right), D$ es el coeficiente de difusión $\left(\mathrm{m}^{2} . \mathrm{s}^{-1}\right)$ y $c$ es la concentración de soluto en el solvente $\left(\mathrm{mol} . \mathrm{m}^{-3}\right)$. Durante la aplicación de la simulación se utilizó una concentración de entrada de $1711 \mathrm{~mol} . \mathrm{m}^{-3}$, una concentración de salida de 0 mol. $\mathrm{m}^{-3}$ y un coeficiente de difusión de $1 \mathrm{~m}^{2} \cdot \mathrm{s}^{-1}$. Se obtuvo una difusión aparente de $0,029 \mathrm{~m}^{2} \cdot \mathrm{s}^{-1}$ (Tabla 1 ).

Posteriormente, sobre la misma porosidad conectada (Figura 6A), se realizó una simulación del factor de formación (Figura 6D) a partir de un algoritmo que resuelve la ley de Ohm (Lemaitre et al., 1988) entre las dos caras opuestas de la muestra digital:

$$
\frac{j \text { total }}{S}=\sigma \frac{\text { Ventrada }- \text { V salida }}{L}
$$

Donde $j$ total es el flujo eléctrico en la cara de entrada, $S$ es el área de la cara de entrada, $V$ entrada y $V$ salida son los potenciales impuestos de entrada y salida y $L$ es la longitud de la muestra.

Durante la ejecución de la prueba se utilizó un voltaje de entrada de $1 \mathrm{~V}$, un voltaje de salida de $0 \mathrm{~V}$ y una conductividad eléctrica de $0,0001 \mathrm{~S} . \mathrm{m}^{-1}$. Se obtuvo un factor de formación de 74,95 (Tabla 1).

\begin{tabular}{|c|c|c|c|c|c|}
\hline \multicolumn{6}{|c|}{ Simulación } \\
\hline \multicolumn{2}{|c|}{ Permeabilidad absoluta } & \multicolumn{2}{|c|}{ Difusión molecular aparente } & \multicolumn{2}{|c|}{ Factor de formación aparente } \\
\hline Presión input & $130.000 \mathrm{~Pa}$ & Concentración input & $\begin{array}{c}1711 \\
\text { mol.m }{ }^{-3}\end{array}$ & Voltaje input & $1 \mathrm{~V}$ \\
\hline Presión output & $100.000 \mathrm{~Pa}$ & Concentración output & $0 \mathrm{~mol} \cdot \mathrm{m}^{-3}$ & Voltaje output & $0 \mathrm{~V}$ \\
\hline Viscosidad del fluido & 0,001 Pa.s & Difusividad bulk & $1 \mathrm{~m}^{2} \cdot \mathrm{s}^{-1}$ & Conductividad eléctrica & 0,0001 S.m ${ }^{-1}$ \\
\hline $\begin{array}{l}\text { Dirección de } \\
\text { simulación }\end{array}$ & $\mathrm{y}$ & $\begin{array}{l}\text { Dirección de } \\
\text { simulación }\end{array}$ & $\mathrm{y}$ & Dirección de simulación & $\mathrm{y}$ \\
\hline K absoluta obtenida & 8,28 e-07 d & $\begin{array}{l}\text { Difusión molecular } \\
\text { aparente }\end{array}$ & $0,029 \mathrm{~m}^{2} \cdot \mathrm{s}^{-1}$ & $\begin{array}{l}\text { Factor de formación } \\
\text { aparente }\end{array}$ & 74,95 \\
\hline
\end{tabular}

Tabla 1. Resultados obtenidos en medio poroso conectado de la muestra \#1 Formación Vaca Muerta.

\section{Caso de estudio: muestra \#2 Formación La Luna}

Durante la revisión del volumen de FIB-SEM de la muestra perteneciente a la Formación La Luna, se identificaron poros inorgánicos y poros relacionados con la materia orgánica, como se observa en la Figura 7. La porosidad de la muestra se concentra en la parte superior del volumen FIB-SEM (Figura 8A) y solo una porción de esta porosidad se encuentra conectada a lo largo del eje Z (Figura 10B).

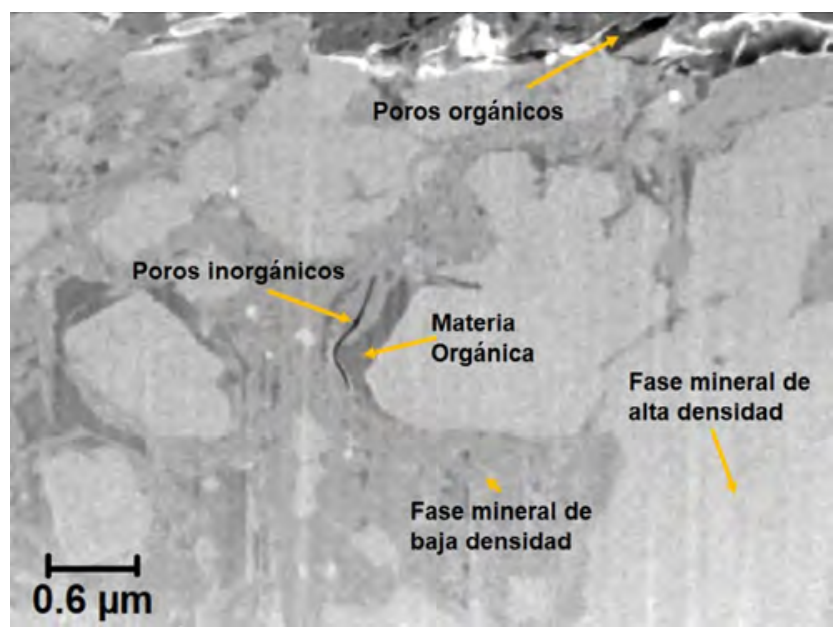

Figura 7. Caracterización cualitativa de imagen FIB-SEM en muestra \#2 Formación La Luna. 
La muestra presentó una porosidad total de 0,4\%, como se muestra en la Tabla 2. Posteriormente, a partir del uso de la porosidad total segmentada (Figura 8A) se realiza una separación de objetos, proceso que hace uso de un algoritmo que combina una segmentación de tipo watershed (Levner y Zhang, 2007) y una transformación de distancia con el fin de dividir píxeles asociados a cada poro. Lo anterior permite obtener la distribución de tamaño de poro presente en la muestra. Se obtuvo una distribución unimodal, con una de moda de $5 \mathrm{~nm}$ (Figura 9). Se identificó porosidad conectada (7,61\%) a lo largo del eje Z (Figura 8B), sobre la cual se aplicó una simulación de permeabilidad absoluta. A partir de una presión de entrada y salida de $130.000 \mathrm{~Pa}$ y $100.000 \mathrm{~Pa}$, respectivamente, se obtuvo una permeabilidad absoluta en dirección $\mathrm{Z}$ sin escalamiento de 0,0362 mD. Una muestra de la Formación La Luna con profundidad coincidente presentó porosidad RCAL (routine core analysis) de 8,2\% y una permeabilidad al aire (RCAL) de $0,0015 \mathrm{mD}$.

Tabla 2. Resultado de la porosidad de la muestra \#2 Formación La Luna.

\begin{tabular}{cccc}
\hline & Fracción Volumétrica & Vóxeles segmentados & Vóxeles totales \\
\hline Porosidad & 0,00402 & 665.166 & 165.306 .752 \\
\hline
\end{tabular}

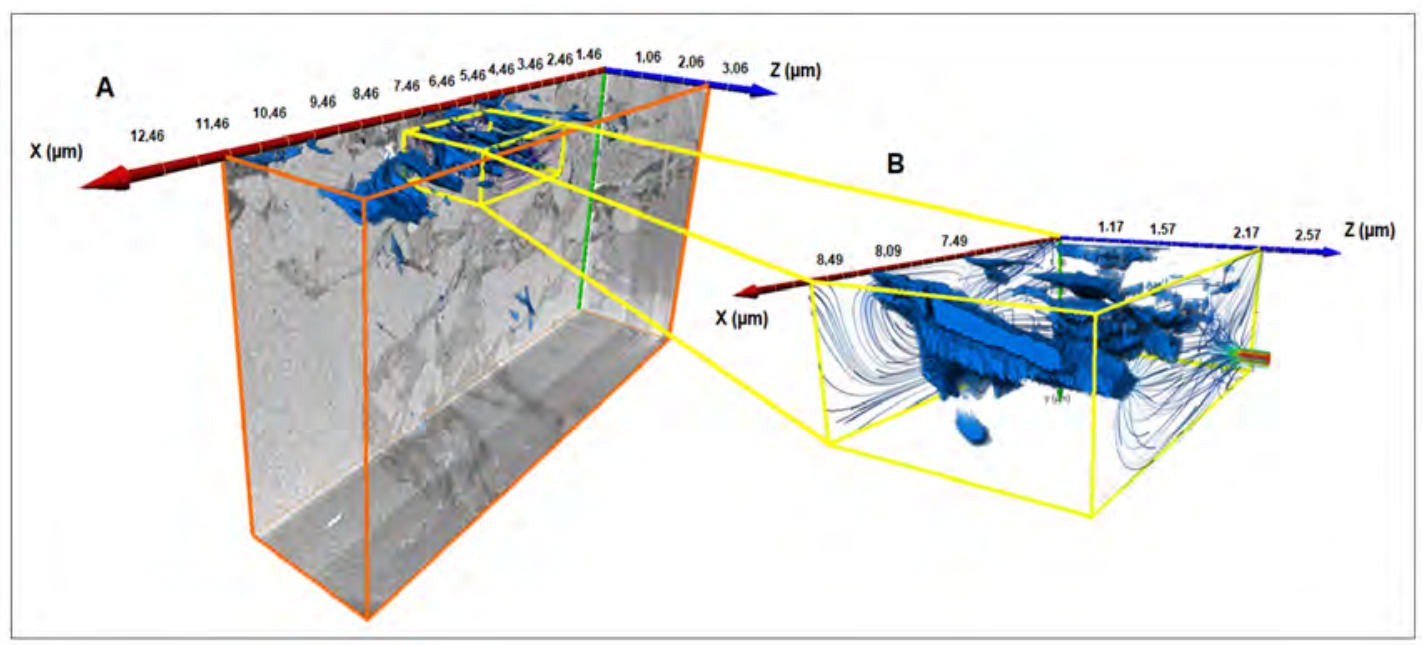

Figura 8. Volumen digital FIB-SEM obtenido en muestra \#2 Formación La Luna. A. Porosidad total presente en la muestra (porosidad total segmentada en color azul). B. Líneas iluminadas representan el campo de velocidad durante la simulación de permeabilidad absoluta realizada en la porosidad conectada de la muestra (porosidad conectada segmentada en color azul dentro del recuadro amarillo).

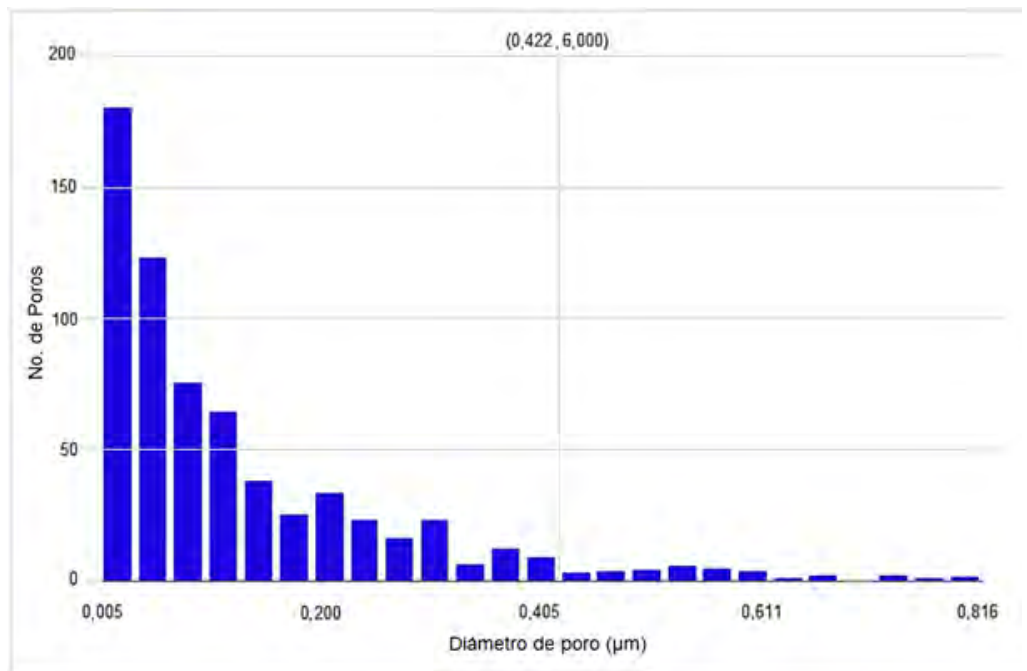

Figura 9. Distribución de tamaño de poro en muestra \#2 Formación La Luna. 
La porosidad conectada a lo largo del eje Z (Figura 8B) permitió ejecutar una simulación de la presión capilar por el método de inyección de mercurio. La simulación calcula la presión capilar a partir de la ecuación de Young-Laplace (Jiao et al., 2020):

$$
P c=\frac{2 \sigma \cos \theta}{\text { radio }}
$$

En donde $\sigma$ es la tensión interfacial mercurio-aire $(485,5 \mathrm{mN} / \mathrm{m})$ y $\theta$ es el ángulo de contacto, que para el caso de la intrusión de mercurio es igual a 0 , ya que se trata de un fluido completamente no mojante.

La presión capilar obtenida con la simulación fue comparada con resultados experimentales a partir del método de laboratorio Mercury Injection Capillary Pressure (MICP) en una muestra de la Formación La Luna con profundidad coincidente. Se observa que los valores obtenidos con la metodología digital son consistentes en tendencia con los resultados experimentales (Figura 10). Sin embargo, la simulación predice presiones capilares un 55,8\% más altas que el método convencional, lo cual puede ocurrir debido a que la muestra digital no alcanza el representative elementary volume (REV), a la presencia de alta heterogeneidad en la zona donde se tomó el volumen digital FIB-SEM, a problemas de segmentación de gargantas de poro por falta de resolución de imagen (poros con diámetro menor a $10 \mathrm{~nm}$ ) y a la falta de escalamiento del dato obtenido.

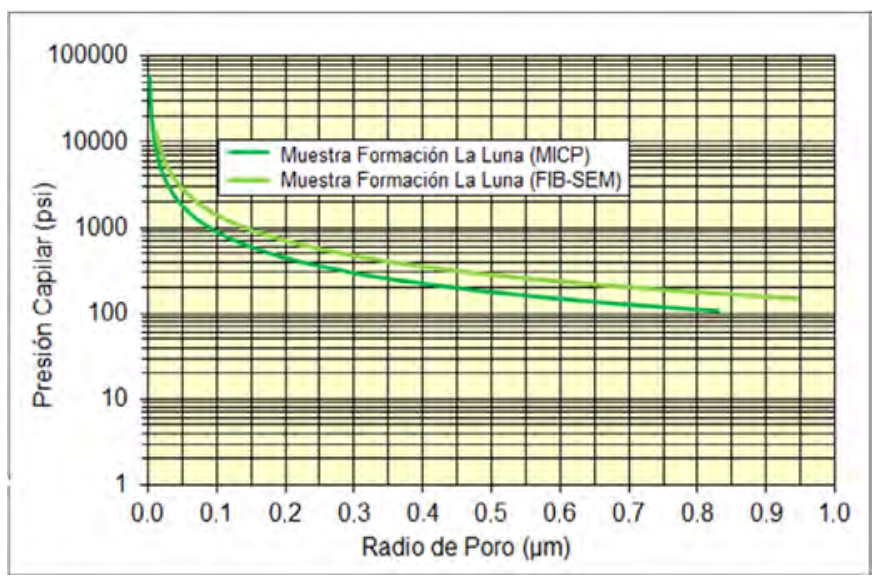

Figura 10. Presión capilar vs radio de poro en la muestra \#2 Formación La Luna a partir del método digital FIB-SEM y convencional de laboratorio MICP.

\section{Estudio comparativo: porosidad y materia orgánica}

Se tomaron 13 imágenes digitales BIB-SEM de diferentes profundidades de la Formación la Luna y se realizó el cálculo de porosidad 2D, el cual fue comparado con resultados de porosidad en tapones siguiendo las técnicas convencionales (RCAL) en profundidades coincidentes. Los resultados obtenidos a partir de las imágenes BIB-SEM muestran valores menores de porosidad en comparación con la porosidad obtenida experimentalmente (Figura 11), lo cual es consistente con la diferencia en escala de las dos técnicas analíticas. Tampoco se observa una tendencia significativa entre la porosidad RCAL y la porosidad BIB-SEM, lo cual está relacionado con la alta heterogeneidad de la muestra analizada.

Posteriormente, se tomaron las mismas 13 imágenes digitales BIB-SEM de diferentes profundidades de la Formación La Luna con el fin de cuantificar el contenido de materia orgánica (bitumen/kerógeno) presente en cada imagen y se realizó la comparación con resultados obtenidos con el método convencional LECO TOC de laboratorio de geoquímica en muestras con profundidades coincidentes. Los resultados en la Figura 12 indican que la materia orgánica cuantificada a partir de imágenes digitales es mayor que la obtenida por el método tradicional. Las diferencias encontradas se deben a la heterogeneidad de las muestras analizadas, ya que no se tienen datos de laboratorio exactamente en la misma profundidad de cada imagen BIB-SEM, a la concentración de materia orgánica de la región de interés en donde se tomaron las imágenes BIBSEM, y a la falta de conversión entre el porcentaje en peso y el porcentaje en volumen, ya que no se tiene la densidad exacta del kerógeno presente en las muestras analizadas. 


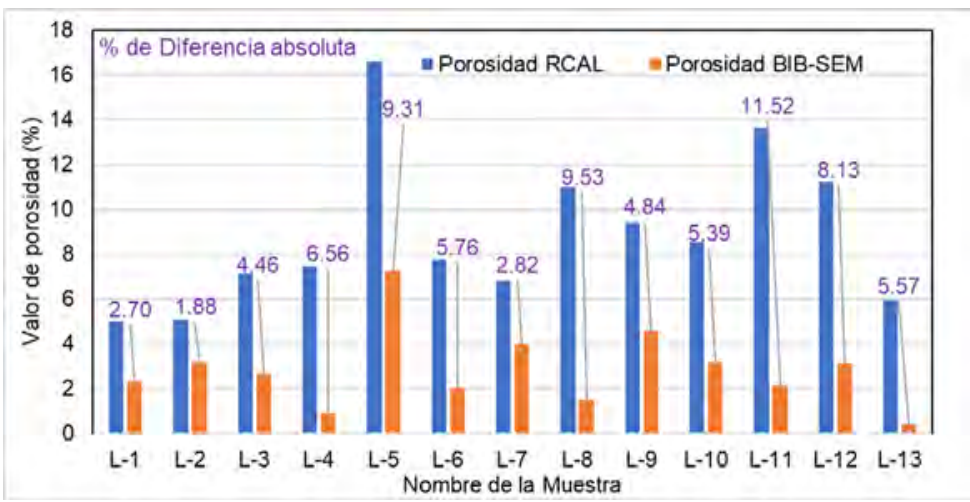

Figura 11. Porosidad a partir de imágenes BIB-SEM vs porosidad RCAL en muestras de la Formación La Luna.

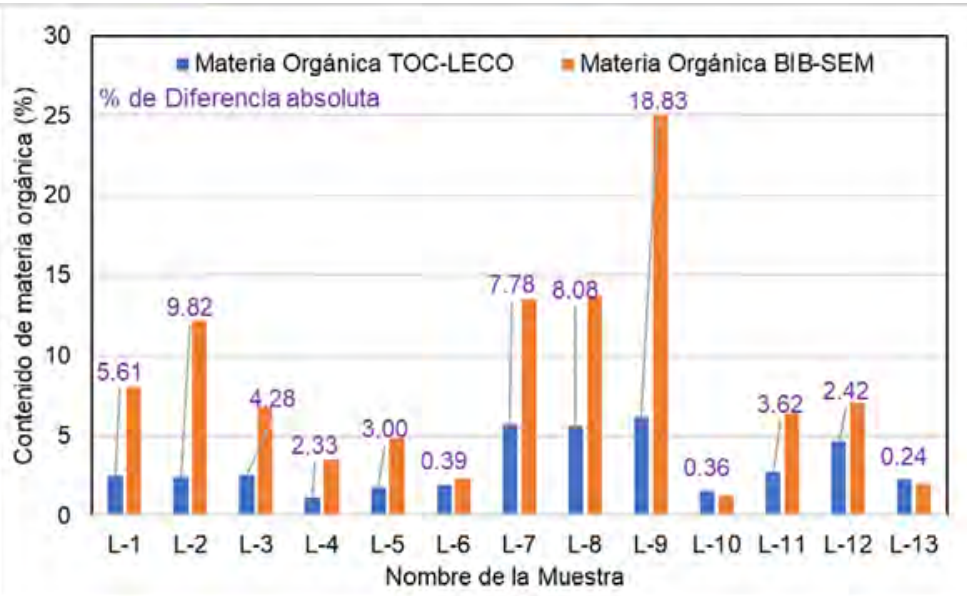

Figura 12. Contenido de materia orgánica a partir de imágenes BIB-SEM vs TOC obtenido experimentalmente en muestras de la Formación La Luna.

\section{Discusión}

Las técnicas digitales (FIB-SEM, BIB-SEM y microCT) complementan la caracterización de muestras de yacimientos no convencionales, ya que permiten definir el tipo de porosidad predominante (porosidad relacionada con la materia orgánica o porosidad interpartícula), identificar el número de fases minerales, cuantificar la cantidad de materia orgánica presente y simular propiedades petrofísicas básicas (porosidad y permeabilidad absoluta) y avanzadas (factor de formación, presión capilar por inyección de mercurio y difusión molecular aparente). Este tipo de técnicas también pueden ser de utilidad en escenarios problemáticos o muy complejos de analizar en los laboratorios convencionales, como es el caso del análisis de muestras naturalmente fracturadas, el cálculo de permeabilidades relativas de tres fases en muestras de baja porosidad y permeabilidad o la medición de propiedades petrofísicas en muestras saturadas por hidrocarburo que requieren diferentes direcciones de desplazamiento.
Al obtener un modelo de matriz mineral y espacio poroso digital de una roca, tapón o muestra de análisis, se eliminan algunas de las limitantes generadas en los equipos tradicionales al intentar inyectar un fluido a través de una muestra (e.g. migración de finos y daño de formación). Sin embargo, la petrofísica digital trae consigo sus propias limitantes que tienen que ser evaluadas al momento de aplicar la técnica (e.g. alto costo del proceso de digitalización, capacidad computacional para simular, representatividad y escalamiento de los resultados obtenidos).

Aunque actualmente se requieren equipos de cómputo muy robustos para ejecutar los algoritmos de simulación sobre modelos de roca digital en alta resolución y con grandes dimensiones (gran tamaño computacional), se espera que, en los años por venir, estas limitantes sean superadas conforme avanza la tecnología computacional. Además, el hecho de tener las rocas digitalizadas trae consigo grandes ventajas que van desde una preservación prolongada en el tiempo hasta la posibilidad de analizar y revisar la 
información rápidamente desde cualquier locación, con la posibilidad de simular diferentes procesos sobre la muestra, inclusive repitiendo el experimento un sinnúmero de veces con parámetros o escenarios completamente distintos y en cualquier dirección deseada, lo cual influye en el estudio de la anisotropía de la roca. Lo anterior es complejo de realizar en un laboratorio convencional, ya que las muestras se almacenan físicamente y se deterioran con el tiempo, algunos equipos solo miden propiedades en una dirección y las pruebas pueden modificar la naturaleza interna de la muestra y, por ende, sus propiedades petrofísicas, por lo que no pueden ser utilizadas en estudios posteriores.

La limitante más importante encontrada al aplicar esta tecnología es la representatividad de los resultados obtenidos: si se trabaja con muestras de roca convencional (areniscas en escala de micras), es posible a través de la técnica de microtomografía obtener rocas digitales que son volumétricamente representativas en alta resolución (tapones completos o incluso núcleos de hasta 1 pie de longitud con la implementación de microtomografía industrial). La tomografía médica (macro-CT) puede escanear un núcleo completo, pero a una resolución muy baja que dificulta definir el medio poroso, incluso en rocas convencionales; sin embargo, esta técnica puede utilizarse para predecir propiedades petrofísicas a partir del número CT, de acuerdo con los lineamientos propuestos por Wellington y Vinegar (1987).

Para el caso de los yacimientos no convencionales, dado que el medio poroso se encuentra en la escala micro- y nanométrica, el instrumento de imagen que puede llegar a visualizar los poros a esa escala es la microscopía electrónica de barrido (FIB-SEM) cuya gran limitante es el restringido volumen digital que produce (cubos de 5 a 60 micras de lado), lo cual da un rango de volumen entre $125 \mu \mathrm{m}^{3}-216.000 \mu \mathrm{m}^{3}$. Además, el hecho de que se trate de un volumen digital muy pequeño no significa que el tamaño computacional de la muestra sea bajo, puesto que existen instrumentos SEM que al tener resoluciones tan altas (menos de $1 \mathrm{~nm}$ por píxel), generan imágenes en extremo pesadas computacionalmente y volúmenes de FIB-SEM del orden de decenas de gigabytes.

Si a lo anterior se agrega la alta heterogeneidad característica de las rocas no convencionales, es bastante claro que el dato obtenido no es representativo del bulk de la roca en cuestión, más bien es un dato que representa una heterogeneidad específica. Por esta razón, es necesario localizar cada una de las heterogeneidades que presenta la muestra, evaluarlas y caracterizarlas con el FIB-SEM en alta resolución (dato no representativo del bulk) y posteriormente escalar la información a modelos más gruesos que se acerquen al mínimo elemental representativo (dato representativo del bulk) a través de la aplicación de metodologías de escalamiento (e.g. análisis multiescala, teoría de la homogenización, reconocimiento y segmentación de texturas de imagen con aprendizaje automático, amarre roca-registro, etc.).

De acuerdo con los resultados obtenidos, en el caso específico de la muestra \#2 perteneciente a la Formación La Luna, se observó alta similitud en la tendencia entre el dato de presión capilar por inyección de mercurio calculado por la técnica digital y el dato obtenido experimentalmente en laboratorio, sin embargo, para el caso de la porosidad y permeabilidad se observaron diferencias absolutas significativas $(7,8 \%$ en porosidad y $0,0347 \mathrm{mD}$ en permeabilidad absoluta), relacionadas principalmente con las diferencias en escala de las técnicas utilizadas y con la heterogeneidad de las muestras analizadas. Por lo tanto, se recomienda enfocar las futuras investigaciones en el desarrollo de metodologías de escalamiento que aprovechen la información obtenida con FIB-SEM en alta resolución (estimación de propiedades petrofísicas a partir de imágenes) para complementar modelos más gruesos obtenidos con micro-CT en baja resolución (distribución de fases minerales, laminaciones, distribución fracturas), los cuales sí son representativos/comparables con los análisis de laboratorio tradicionales.

\section{Agradecimientos}

Los autores desean agradecer a Ecopetrol por el suministro de la estación de trabajo y muestras digitales (FIB-SEM y BIB-SEM) de la Formación La Luna, licenciamiento de software de manejo de imágenes y permiso para publicar los resultados de este estudio.

\section{Referencias}

Al-Amri, S.S.; Kalyankar, N.V.; Khamitkar, S.D. (2010). Image segmentation by using threshold techniques. Journal of Computing, 2(5). 
Andrew, M. (2019). Vaca Muerta FIB-SEM. www. digitalrocksportal.org

Cantisano, M.T.; Restrepo, D.P.; Cespedes, S.; Toelke, J.; Grader, A.; Suhrer, M.; Walls, J. (2013). Relative permeability in a shale formation in Colombia using digital rock physics. Unconventional Resources Technology Conference. Denver, Colorado. https:// doi.org/10.1190/urtec2013-092

Chorin, A.J. (1967). A numerical method for solving incompressible viscous flow problems. Journal of Computational Physics, 2(1), 12-26. https://doi. org/10.1016/0021-9991(67)90037-X

Dvorkin, J.; Derzhi, N.; Fang, Q.; Nur, A., Nur, B.; Grader, A.; Baldwin, C.; Tono, H.; Diaz, E. (2009). From micro to reservoir scale: Permeability from digital experiments. The Leading Edge, 28(12), 1446-1452. https://doi.org/10.1190/1.3272699

Giannuzzi, L.A. (2004). Introduction to focused ion beams: instrumentation, theory, techniques and practice. Springer Science \& Business Media.

Jiao, L.; Andersen, P.Ø.; Zhou, J.; Cai, J. (2020). Applications of mercury intrusion capillary pressure for pore structures: A review. Capillarity, 3(4), 6274. https://doi.org/10.46690/capi.2020.04.02

Klaver, J.; Desbois, G.; Urai, J.; Littke, R. (2012). BIB-SEM study of the pore space morphology in early mature Posidonia Shale from the Hils area, Germany. International Journal of Coal Geology, 103, 12-25. https://doi.org/10.1016/j. coal.2012.06.012

Lemaitre, J.; Troadec, J.P.; Bideau, D.; Gervois, A.; Bougault, E. (1988). The formation factor of the pore space of binary mixtures of spheres. Journal of Physics D: Applied Physics, 21(11), 1589-1592. https://doi.org/10.1088/0022-3727/21/11/007

Levner, I.; Zhang, H. (2007). Classification-driven watershed segmentation. IEEE Transactions on Image Processing, 16(5), 1437-1445. https://doi. org/10.1109/TIP.2007.894239

Li, C.; Wang, D.; Kong, L. (2021). Application of machine learning techniques in mineral classification for scanning electron microscopy-energy dispersive X-Ray spectroscopy (SEM-EDS) Images. Journal of Petroleum Science and Engineering, 200. https:// doi.org/10.1016/j.petrol.2020.108178

Lin, C.L.; Miller, J.D.; Hsieh, C.H.; Tiwari, P.; Deo, M.D. (2010). Pore scale analysis of oil shale pyrolysis by X-ray CT and LB simulation. $6^{\text {th }}$ World Congress on Industrial Process Tomography. Beijing, China.

McLean, R.; Miller, C.; Guzman, B.; Walls, J. (2017). Quantifying organic porosity and predicting estimated ultimate recovery (EUR) in the Eagle Ford Formation. Unconventional Resources Technology Conference. Austin, Texas.

Nozad, I.; Carbonell, R.G.; Whitaker, S. (1985). Heat conduction in multiphase systems-I: theory and experiment for two-phase systems. Chemical Engineering Science, 40(5), 843-855. https://doi. org/10.1016/0009-2509(85)85037-5

Saif, T.; Lin, Q.; Bijeljic, B.; Blunt, M. (2017). Microstructural imaging and characterization of oil shale before and after pyrolysis. Fuel, 197, 562-574. https://doi.org/10.1016/j.fuel.2017.02.030

van Milligen, B.P.; Bons, P.D.; Carreras, B.A.; Sanchez, R. (2005). On the applicability of Fick's law to diffusion in inhomogeneous systems. European Journal of Physics, 26(5), 913-925. https://doi. org/10.1088/0143-0807/26/5/023

Wellington, S.L.; Vinegar, H.J. (1987). X-ray computerized tomography. Journal of Petroleum Technology, 39(08), 885-898. https://doi. org/10.2118/16983-PA

Zhang, L.; Jing, W.; Yang, Y.; Yang, H.; Guo, Y.; Sun, H.; Zhao, J.; Yao, J. (2019). The investigation of permeability calculation using digital core simulation technology. Energies, 12(17). https://doi. org/10.3390/en12173273

Zoback, M.D.; Kohli, A.H. (2019). Unconventional Reservoir Geomechanics. Cambridge University Press.

Fecha de recibido: 21 de febrero de 2020 Fecha de aprobado: 25 de noviembre de 2021 